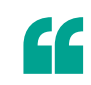

...slither

swimming

itself could

be employed

to separate

sperm with

special

qualities...

\title{
Just keep swimming - sperm exhibit a 2D slither motion
}

New research published in Nature

Communications has shown that human sperm exhibit a distinct 2D 'slither' swimming mode when the entire length of the cell is confined within $1 \mu \mathrm{m}$ of a surface, which is different from the swimming mode observed in 3D bulk swimming.

David Sinton, corresponding author, told Nature Reviews Urology "We were motivated to investigate the exact form of sperm locomotion at surfaces when observing sperm behaviour in our microchannel-based sperm sorting work."

Using total internal reflection fluorescence microscopy and epifluorescence, lead author
Reza Nosrati imaged sperm both away from and within $1 \mu \mathrm{m}$ of a surface and observed that human sperm swim $\sim 50 \%$ faster than bulk swimmers when swimming in the slither mode. The tendency of human sperm to slither swim increased with viscosity. Slither swimming was found to be intermittent, lasting $\sim 1 \mathrm{~s}$, during which the sperm travelled $\sim 70 \mu \mathrm{m}$.

Sinton explained "Regarding the clinical relevance of these observations, the most obvious and immediate implication is in the design of sperm separation and selection devices. We are interested in how - and if - slither swimming itself could be employed to separate

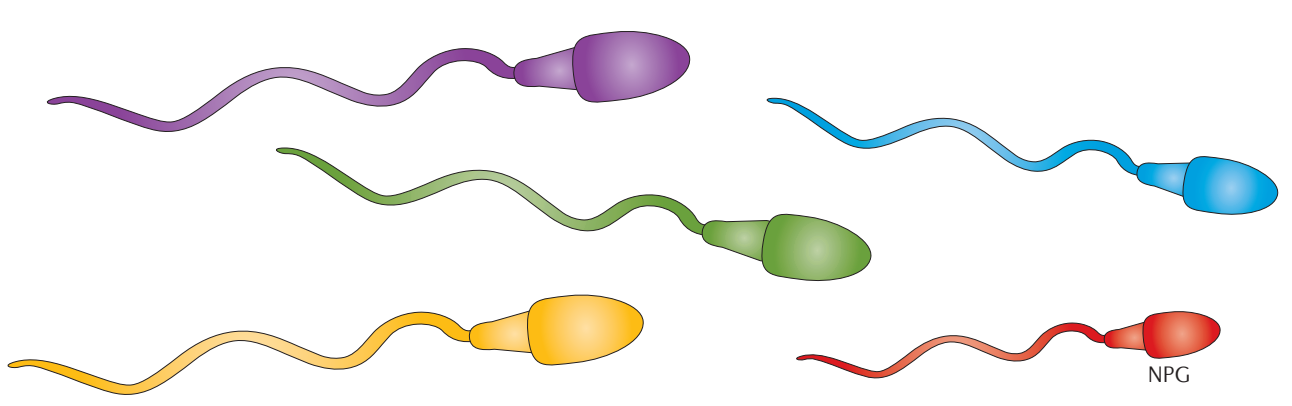

sperm with special qualities. The fact that slither swimming appears to be a preferred way to navigate highly viscous regions of the female reproductive tract makes it an interesting candidate for sperm selection." He continued: "We are currently testing methods to separate this population and assess their characteristics, notably DNA integrity, motility and morphology."

Sinton and colleagues are keen to translate this research into clinical practice and are currently working with urologists and clinical experts to develop these separation technologies. He concluded: "We are hopeful that we can establish a second generation of sperm selection devices that are simple, cheap, and considerably outperform current methods in terms of sperm quality."

Louise Stone

ORIGINAL ARTICLE Nosrati, R. et al.

Two-dimensional slither swimming of sperm within a micrometre of a surface. Nat. Commun. http://dx.doi.org/10.1038/ncomms9703 